\title{
Cooperative TDD reciprocity calibration with diversity combining for distributed large-scale MIMO systems
}

\author{
Hao Wei ${ }^{1,2}$, Dongming Wang ${ }^{1 *}$, Jingyu Hua ${ }^{3}$, Junhui Zhao ${ }^{4}$ \\ 1. National Mobile Communications Research Laboratory, Southeast University, Nanjing 210096, China \\ 2. Zhongxing Telecommunication Equipment Corporation, Shanghai 201203, China \\ 3. Zhejiang University of Technology, Hangzhou 310023, China \\ 4. School of Electronic and Information Engineering, Beijing Jiaotong University, Beijing 100044, China \\ * Corresponding author, Email: wangdm@seu.edu.cn
}

\begin{abstract}
Thanks to channel reciprocity, the time-division duplexing operation is typically used for distributed large-scale multiple-input multiple-output systems. With the knowledge of uplink channel state, APs (Access Points) perform the downlink precoding to cooperatively communicate with multiple UEs (User Equipments). Unfortunately, the channel reciprocity is often jeopardized by the non-symmetric transceiver RF (Radio Frequency) circuits at both sides of the link. By excluding UEs from the calibration procedure, the TLS (Total Least Squares) method is extended to the case of partial calibration, where only the APs are involved to exchange calibration signals to compensate for RF mismatches. Nevertheless, channel fluctuations between APs significantly degrade system performance. Therefore, we propose a scheme to obtain the diversity gain, by performing a calibration with the combination of the calibration signals of different channel coherent times. Simulation results confirm the effective performance and robustness of our scheme.
\end{abstract}

Keywords: reciprocity calibration, RF mismatch, large-scale MIMO, diversity combining, time-division duplexing

Citation: H. Wei, D. M. Wang, J. Y. Hua, et al. Cooperative TDD reciprocity calibration with diversity combining for distributed large-scale MIMO systems [J]. Journal of communications and information networks, 2017, 2(3): 61-67.

\section{Introduction}

For an innovative scaling up, a large number of antennas are equipped at a transmitter to improve spectral efficiency and energy efficiency. This is known as massive MIMO (Multiple-Input MultipleOutput) or large-scale MIMO technology ${ }^{[1-3]}$. In distributed MIMO systems, large-scale antennas are located at some APs. A central processing unit connecting these distributively deployed APs through

Manuscript received May 30, 2016; accepted Nov. 11, 2016

This work is supported in part by the National Basic Research Program of China ("973" Program) (No. 2013CB336600); the Natural Science Foundation of China (NSFC) (Nos. 61271205, 61501113, 61521061, 61372100); National High Technology Research and Development Program of China ("863" Program) (No. 2014AA01A704); the Natural Science Foundation of Jiangsu Province (No. BK20150630); the Hong Kong, Macao and Taiwan Science \& Technology Cooperation Program of China (No. 2014DFT10290); and the Fundamental Research Funds for the Central Universities and Colleges and Universities in Jiangsu Province Plans to Graduate Research and Innovation (No. KYLX15_0075). 
high-bandwidth wire links ${ }^{[4,5]}$. Given the downlink CSI (Channel State Information), the APs can perform joint precoding to cooperatively communicate with multiple UEs on a single time-frequency resource. In a TDD (Time-Division Duplexing) operation, an estimation of the uplink CSI can be seen as the transpose of the downlink CSI thanks to the channel reciprocity. Thus, the transmitter can estimate the CSI for both uplink and downlink channels only through uplink pilots from the UEs, which saves large resources since the orthogonal downlink pilots from the APs and the CSI feedback from UEs are not required $^{[2]}$. Unfortunately, transceiver $\mathrm{RF}$ circuits at both sides of the link are usually not symmetrical. Since the transmitting signals also experience the RF circuits, the RF mismatches will jeopardize the reciprocity of the entire channel and can lead to a severe loss in system performance ${ }^{[6]}$. Therefore, it is very important to perform a reciprocity calibration.

In order to compensate for the RF mismatches, a calibration scheme named the TLS method was proposed in Ref. [7], which formed the calibration as a TLS problem. However, by the TLS method, the calibration signals need to be exchanged between the transmitters and the receivers. Feedback from the receivers is also required, and will cause heavy overhead in large-scale MIMO systems. Fortunately, the RF mismatches at the receivers can be ignored since they have a negligible influence on system performance $^{[8]}$. Thus, only a partial calibration was necessary to compensate for the RF mismatches at the transmitters ${ }^{[9]}$. For distributed systems, the LS (Least Squares) method was presented in Ref. [10]. By avoiding the involvement of UEs, the LS method requires only the sequential exchanging of calibration signals between APs. The LS method has been proven as an extension of the TLS method to the case of self-calibration ${ }^{[11]}$.

Nevertheless, in a distributed topology, the channels between APs are usually random (and are often Rayleigh distributed). Thus, the SNR (Signal-toNoise Ratio) of the received calibration signals may not be large. This quick fluctuation will significantly degrade system performance. In this paper, we pro- pose a scheme to perform the calibration in different channel coherent times. Then, we calculate the calibration coefficients according to a reconstructed LS function using a combination of the calibration signals. Owing to the diversity gain, system performance and robustness can be greatly improved.

Notations Vectors are denoted in lower case bold: $\boldsymbol{x}$. Matrices are in upper case bold: $\boldsymbol{A}$. $[\boldsymbol{A}]_{i, j}$ denotes the $i$ th row $j$ th column element of $\boldsymbol{A} .(\cdot)^{*},(\cdot)^{\mathrm{T}}$ and $(\cdot)^{\mathrm{H}}$ represent the conjugate, transpose, and Hermitian transpose, respectively. $\operatorname{diag}(\boldsymbol{x})$ is a diagonal matrix with $\boldsymbol{x}$ on its diagonal, and $\operatorname{diag}(\boldsymbol{A})$ denotes a column vector with the main diagonal of $\boldsymbol{A}$.

\section{System model of transceiver RF circuits}

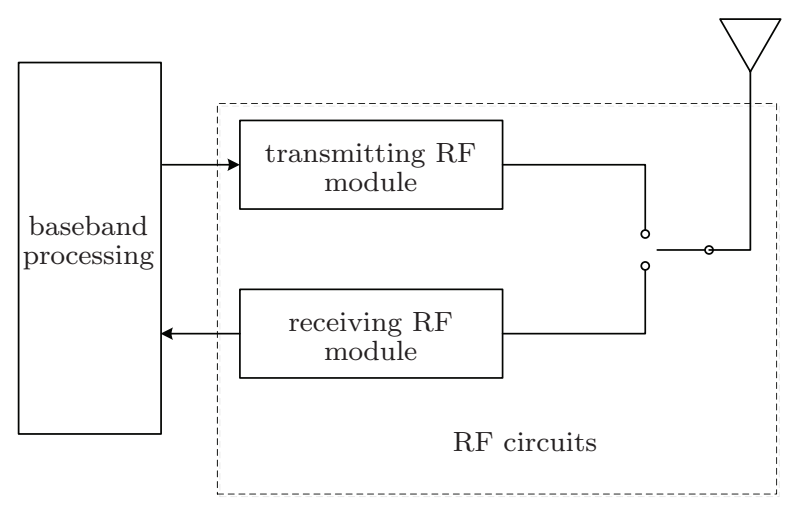

Figure 1 Model of RF circuits

In practice, transceiver RF circuits at both sides of the link are not symmetric owing to variations in the RF and baseband hardware. As shown in Fig. 1, each antenna has a transmitting $\mathrm{RF}$ and a receiving $\mathrm{RF}$ module. $\mathrm{RF}$ circuits include the antennas, mixers, filters, A/D (Analog to Digital) converters, power amplifiers, etc., and are strongly related to the temperature and humidity of the environment ${ }^{[7]}$. It is assumed that there are $M$ antennas in total at the APs and $K$ antennas at the UEs. Denoting $\boldsymbol{H}$ as the wireless downlink channel matrix, the entire uplink and downlink channel matrices are given by

$$
\begin{gathered}
\boldsymbol{G}_{\mathrm{UL}}=\boldsymbol{C}_{\mathrm{AP}, \mathrm{r}} \boldsymbol{H}^{\mathrm{T}} \boldsymbol{C}_{\mathrm{UE}, \mathrm{t}}, \\
\boldsymbol{G}_{\mathrm{DL}}=\boldsymbol{C}_{\mathrm{UE}, \mathrm{r}} \boldsymbol{H} \boldsymbol{C}_{\mathrm{AP}, \mathrm{t}} .
\end{gathered}
$$


$\boldsymbol{C}_{\mathrm{AP}, \mathrm{t}}$ and $\boldsymbol{C}_{\mathrm{AP}, \mathrm{r}}$ denote the transmitting and receiving $\mathrm{RF}$ gain matrices corresponding to the antennas of the APs, respectively. $\boldsymbol{C}_{\mathrm{UE}, \mathrm{t}}$ and $\boldsymbol{C}_{\mathrm{UE}, \mathrm{r}}$ denote the transmitting and receiving $\mathrm{RF}$ gain matrices corresponding to the antennas of the UEs, respectively. Define

$$
\begin{aligned}
& \boldsymbol{C}_{\mathrm{AP}, \mathrm{t}}=\operatorname{diag}\left(t_{\mathrm{AP}, 1}, \cdots, t_{\mathrm{AP}, m}, \cdots, t_{\mathrm{AP}, M}\right), \\
& \boldsymbol{C}_{\mathrm{AP}, \mathrm{r}}=\operatorname{diag}\left(r_{\mathrm{AP}, 1}, \cdots, r_{\mathrm{AP}, m}, \cdots, r_{\mathrm{AP}, M}\right), \\
& \boldsymbol{C}_{\mathrm{UE}, \mathrm{t}}=\operatorname{diag}\left(t_{\mathrm{UE}, 1}, \cdots, t_{\mathrm{UE}, k}, \cdots, t_{\mathrm{UE}, K}\right), \\
& \boldsymbol{C}_{\mathrm{UE}, \mathrm{r}}=\operatorname{diag}\left(r_{\mathrm{UE}, 1}, \cdots, r_{\mathrm{UE}, k}, \cdots, r_{\mathrm{UE}, K}\right),
\end{aligned}
$$

where $t_{\mathrm{AP}, m}, r_{\mathrm{AP}, m}(m=1, \cdots, M)$ are the $\mathrm{RF}$ gains of the $m$ th antenna at the APs, and $t_{\mathrm{UE}, k}$, $r_{\mathrm{UE}, k}(k=1, \cdots, K)$ are the RF gains of the $k$ th antenna at the UEs. It can be seen from Eqs. (1) and (2) that the uplink and downlink channels become non-reciprocal owing to the RF mismatches. Thus, it turns out to be $\boldsymbol{G}_{\mathrm{DL}} \neq \boldsymbol{G}_{\mathrm{UL}}^{\mathrm{T}}$, although the wireless channels are symmetrical.

\section{Cooperative TDD reciprocity cali- bration}

\subsection{TLS method with partial calibration}

The TLS method forms a calibration model by a TLS problem, which demands the exchange of calibration signals between the transmitters and receivers ${ }^{[7]}$. To avoid involving the UEs in the calibration process, the TLS method was extended to the case of the partial calibration. In Ref. [11], the TLS method with partial calibration was proved equivalent to the LS method in Ref. [10]. Only the sequential exchange of calibration signals between APs is required.

During the calibration procedure, time-orthogonal pilot symbols are exchanged by the antennas of the APs with power $P_{\text {cal }}$. For clarity of discussion, the value of the calibration signal is set to be 1 . Then, the received calibration signal matrix is given by

$$
\boldsymbol{Y}_{\text {cal }}=\boldsymbol{C}_{\mathrm{AP}, \mathrm{r}} \boldsymbol{H}_{\mathrm{cal}} \boldsymbol{C}_{\mathrm{AP}, \mathrm{t}}+\boldsymbol{N},
$$

where

$$
\left[\boldsymbol{Y}_{\text {cal }}\right]_{m, n}= \begin{cases}r_{\mathrm{AP}, m}\left[\boldsymbol{H}_{\mathrm{cal}}\right]_{m, n} t_{\mathrm{AP}, n}+[\boldsymbol{N}]_{m, n}, & m \neq n, \\ 0, & m=n\end{cases}
$$

is the calibration signal transmitted from the $n$th antenna of the APs to the $m$ th antenna of the APs. $[\boldsymbol{N}]_{m, n}$ is the corresponding equivalent AWGN (Additive White Gaussian Noise) with zero mean and variance $\sigma_{\mathrm{n}}^{2} . \boldsymbol{H}_{\text {cal }}$ is the wireless calibration channel matrix between APs, where $\left[\boldsymbol{H}_{\text {cal }}\right]_{m, n}$ denotes the wireless channel coefficient between the $n$th antenna of the APs and the $m$ th antenna of the APs, and $\left[\boldsymbol{H}_{\mathrm{cal}}\right]_{m, n}=0$ when $m=n$. Given the reciprocity of the wireless channel, we obtain the relationship $\boldsymbol{H}_{\text {cal }}=\boldsymbol{H}_{\text {cal }}^{\mathrm{T}}$. Define the perfect partial calibration matrix as ${ }^{[11]}$

$$
\boldsymbol{C}_{\mathrm{cal}}=\alpha_{\mathrm{cal}} \boldsymbol{C}_{\mathrm{AP}, \mathrm{t}}^{-1} \boldsymbol{C}_{\mathrm{AP}, \mathrm{r}},
$$

where $\alpha_{\text {cal }}$ is a positive scalar that can be eliminated with normalized transmitting power. If the thermal noise is ignored for the received observation matrix $\boldsymbol{Y}_{\text {cal }}$ in Eq. (3), we obtain the following equation

$$
\boldsymbol{Y}_{\mathrm{cal}} \boldsymbol{C}_{\mathrm{cal}}=\boldsymbol{C}_{\mathrm{cal}} \boldsymbol{Y}_{\mathrm{cal}}^{\mathrm{T}} \text {. }
$$

While in the presence of observation noise, $\boldsymbol{C}_{\text {cal }}$ can be obtained by solving an optimization problem as follows

$$
\underset{\left\{\boldsymbol{C}_{\text {cal }}\right\}}{\arg \min }\left\|\operatorname{vec}\left(\boldsymbol{Y}_{\text {cal }} \boldsymbol{C}_{\text {cal }}\right)-\operatorname{vec}\left(\boldsymbol{C}_{\text {cal }} \boldsymbol{Y}_{\text {cal }}^{\mathrm{T}}\right)\right\|^{2} .
$$

Then, we transform the diagonal calibration matrix into a vector as

$$
\boldsymbol{c}_{\text {cal }}=\operatorname{diag}\left(\boldsymbol{C}_{\text {cal }}\right)=\left[c_{1}, \cdots, c_{m}, \cdots, c_{M}\right]^{\mathrm{T}} .
$$

From the statement in Refs. [11,12], Eq. (11) is equivalent to the following unconstrained optimization problem

$$
\min f\left(\boldsymbol{c}_{\mathrm{cal}}\right)=\frac{\boldsymbol{c}_{\mathrm{cal}}^{\mathrm{H}} \boldsymbol{A} \boldsymbol{c}_{\mathrm{cal}}}{\boldsymbol{c}_{\mathrm{cal}}^{\mathrm{H}} \boldsymbol{c}_{\mathrm{cal}}},
$$

where the elements of $\boldsymbol{A}$ are given by

$$
[\boldsymbol{A}]_{i, j}=\left\{\begin{array}{cc}
\sum_{u=1, u \neq i}^{M}\left|\left[\boldsymbol{Y}_{\mathrm{cal}}\right]_{u, i}\right|^{2}, & i=j, \\
-\left[\boldsymbol{Y}_{\mathrm{cal}}\right]_{j, i}^{*}\left[\boldsymbol{Y}_{\mathrm{cal}}\right]_{i, j}, & i \neq j,
\end{array}\right.
$$


According to the matrix principle of the Rayleigh quotient, we have

$$
\lambda_{\min } \leqslant \frac{c_{\mathrm{cal}}^{\mathrm{H}} \boldsymbol{A} \boldsymbol{c}_{\mathrm{cal}}}{\boldsymbol{c}_{\mathrm{cal}}^{\mathrm{H}} \boldsymbol{c}_{\mathrm{cal}}} \leqslant \lambda_{\max },
$$

where $\lambda_{\min }$ and $\lambda_{\max }$ denote the minimum and maximum eigenvalue of $\boldsymbol{A}$, respectively. Thus, the solution for $\boldsymbol{c}_{\text {cal }}$ is the eigenvector of $\boldsymbol{A}$ corresponding to $\lambda_{\min }$.

\subsection{TLS method with diversity combin- ing}

For the TLS method, the calibration procedure only requires the APs to exchange calibration signals and is transparent to the UEs. However, in a distributed topology, the channels between APs usually fluctuate rapidly. The SNR of the received calibration signals may not be large. Thus, some calibration coefficients may be estimated incorrectly, which will distort the multi-user MIMO precoding and result in a significant degradation in performance. Fortunately, the RF gain is stable over long periods of time such as several hours ${ }^{[8,13]}$, which is much longer than the wireless channel coherence time. Thus, we can perform a calibration procedure several times in different channel coherent slots. Then, the diversity gain can be achieved by combining the multiple versions of the calibration signals.

Using our scheme, the calibration procedures are performed in $Q$ different channel coherent slots where RF gains are considered to be constant. Then, exploiting the multiple versions of calibration signals, the optimization problem in Eq. (11) can be reconstructed as

$$
\underset{\left\{\boldsymbol{C}_{\text {cal }}\right\}}{\arg \min } \sum_{q=1}^{Q}\left\|v e c\left(\boldsymbol{Y}_{\text {cal }}^{(q)} \boldsymbol{C}_{\text {cal }}\right)-v e c\left(\boldsymbol{C}_{\text {cal }} \boldsymbol{Y}_{\text {cal }}^{(q)}\right)\right\|^{2},
$$

where $\boldsymbol{Y}_{\text {cal }}^{(q)}$ is the received calibration signal matrix in the $q$ th calibration slot. Then, by the similar derivation in the above subsection, we can obtain the following optimization problem with diversity combining as

$$
\min \quad f_{Q}\left(\boldsymbol{c}_{\mathrm{cal}}\right)=\frac{\boldsymbol{c}_{\mathrm{cal}}^{\mathrm{H}} \boldsymbol{A}_{Q} \boldsymbol{c}_{\mathrm{cal}}}{\boldsymbol{c}_{\mathrm{cal}}^{\mathrm{H}} \boldsymbol{c}_{\mathrm{cal}}},
$$

where

$$
\boldsymbol{A}_{Q}=\sum_{q=1}^{Q} \boldsymbol{A}^{(q)}
$$

The elements of $\boldsymbol{A}^{(q)}$ are given by

$$
\left[\boldsymbol{A}^{(q)}\right]_{i, j}=\left\{\begin{array}{c}
\sum_{u=1, u \neq i}^{M}\left|\left[\boldsymbol{Y}_{\text {cal }}^{(q)}\right]_{u, i}\right|^{2}, \quad i=j, \\
-\left[\boldsymbol{Y}_{\text {cal }}^{(q)}\right]_{j, i}^{*}\left[\boldsymbol{Y}_{\text {cal }}^{(q)}\right]_{i, j}, \quad i \neq j .
\end{array}\right.
$$

Thus, the elements of $\boldsymbol{A}_{Q}$ are written as

$$
\left[\boldsymbol{A}_{Q}\right]_{i, j}= \begin{cases}\sum_{q=1}^{Q} \sum_{u=1, u \neq i}^{M}\left|\left[\boldsymbol{Y}_{\mathrm{cal}}^{(q)}\right]_{u, i}\right|^{2}, & i=j, \\ -\sum_{q=1}^{Q}\left[\boldsymbol{Y}_{\mathrm{cal}}^{(q)}\right]_{j, i}^{*}\left[\boldsymbol{Y}_{\mathrm{cal}}^{(q)}\right]_{i, j}, & i \neq j .\end{cases}
$$

Consequently, by the TLS method with diversity combining, the solution for $\boldsymbol{c}_{\mathrm{cal}}$ is the eigenvector corresponding to the minimum eigenvalue of $\boldsymbol{A}_{Q}$.

\subsection{Analysis for diversity gain}

In this subsection, we will perform the analysis for diversity gain using our proposed calibration scheme. For clarity of discussion, if the thermal noise is not considered, then the elements of $\boldsymbol{A}$ in Eq. (14) can be rewritten as

$$
[\boldsymbol{A}]_{i, j}= \begin{cases}\sum_{u=1, u \neq i}^{M}\left|r_{\mathrm{AP}, u}\right|^{2}\left|t_{\mathrm{AP}, i}\right|^{2}\left|\left[\boldsymbol{H}_{\mathrm{cal}}\right]_{u, i}\right|^{2}, & i=j, \\ -r_{\mathrm{AP}, j}^{*} r_{\mathrm{AP}, i} t_{\mathrm{AP}, i}^{*} t_{\mathrm{AP}, j}\left|\left[\boldsymbol{H}_{\mathrm{cal}}\right]_{i, j}\right|^{2}, & i \neq j .\end{cases}
$$

Similarly, the elements of $\boldsymbol{A}_{Q}$ are reformed as

$$
\left[\boldsymbol{A}_{Q}\right]_{i, j}= \begin{cases}\sum_{u=1, u \neq i}^{M}\left|r_{\mathrm{AP}, u}\right|^{2}\left|t_{\mathrm{AP}, i}\right|^{2}\left[\boldsymbol{H}_{\mathrm{cal}}^{Q}\right]_{u, i}, & i=j, \\ -r_{\mathrm{AP}, j}^{*} r_{\mathrm{AP}, i} t_{\mathrm{AP}, i}^{*} t_{\mathrm{AP}, j}\left[\boldsymbol{H}_{\mathrm{cal}}^{Q}\right]_{i, j}, & i \neq j,\end{cases}
$$

where the elements of $\boldsymbol{H}_{\text {cal }}^{Q}$ are

$$
\left[\boldsymbol{H}_{\mathrm{cal}}^{Q}\right]_{i, j}=\sum_{q=1}^{Q}\left|\left[\boldsymbol{H}_{\mathrm{cal}}^{(q)}\right]_{i, j}\right|^{2} .
$$

Then, comparing Eq. (21) with Eq. (22), the TLS method with diversity combining can achieve a diversity gain of $Q$. 


\subsection{Achievable system sum-rates}

In this letter, zero-forcing precoding is used for largescale MIMO systems. Before transmitting the signals, we multiply the precoding matrix by the calibration matrix derived above. Considering the calibration error caused by the observation noise, the calibration matrix is ${ }^{[6]}$

$$
C_{\text {cal }}=C_{\zeta} C_{\mathrm{AP}, \mathrm{t}}^{-1} \boldsymbol{C}_{\mathrm{AP}, \mathrm{r}},
$$

where $\boldsymbol{C}_{\zeta}$ is the diagonal calibration error matrix. Then, ignoring the estimation error of the uplink CSI, the overall downlink received signals at the UEs are given by

$$
\boldsymbol{y}=\beta \boldsymbol{G}_{\mathrm{DL}} \boldsymbol{C}_{\mathrm{cal}} \boldsymbol{G}_{\mathrm{UL}}^{*}\left(\boldsymbol{G}_{\mathrm{UL}}^{\mathrm{T}} \boldsymbol{G}_{\mathrm{UL}}^{*}\right)^{-1} \boldsymbol{x}+\boldsymbol{n},
$$

where $\beta$ is the scaling factor to satisfy the transmit power constraint, $\boldsymbol{y}=\left[y_{1}, \cdots, y_{K}\right]^{\mathrm{T}}$ denotes the received signal vector, $\boldsymbol{x}=\left[x_{1}, \cdots, x_{K}\right]^{\mathrm{T}}$ denotes the signal vector transmitted to the UEs with the power constraint $\mathcal{E}\left[x_{k} x_{k}^{*}\right]=P$, and $\boldsymbol{n}$ is the complex AWGN vector, in which the elements are independent and identically distributed (i.i.d.) complex Gaussian random variables with zero mean and variance $\sigma_{\mathrm{n}}^{2}$. Substituting Eqs. (1) and (2) into Eq. (25), we obtain

$$
\boldsymbol{y}=\beta \boldsymbol{C}_{\mathrm{UE}, \mathrm{r}} \boldsymbol{W}_{\zeta} \boldsymbol{C}_{\mathrm{UE}, \mathrm{t}}^{-1} \boldsymbol{x}+\boldsymbol{n},
$$

where

$$
\begin{aligned}
\boldsymbol{W}_{\zeta}= & \left(\boldsymbol{H} \boldsymbol{C}_{\zeta} \boldsymbol{C}_{\mathrm{AP}, \mathrm{r}} \boldsymbol{C}_{\mathrm{AP}, \mathrm{r}}^{*} \boldsymbol{H}^{\mathrm{H}}\right) \\
& \times\left(\boldsymbol{H} \boldsymbol{C}_{\mathrm{AP}, \mathrm{r}} \boldsymbol{C}_{\mathrm{AP}, \mathrm{r}}^{*} \boldsymbol{H}^{\mathrm{H}}\right)^{-1} .
\end{aligned}
$$

It can be seen from Eq. (27) that, $\boldsymbol{W}_{\zeta}$ is not an identity matrix because of the calibration error. Thus, the received signal of the $i$ th $\mathrm{UE}$ is

$$
\begin{aligned}
y_{i}= & \beta\left[\boldsymbol{W}_{\zeta}\right]_{i, i} \frac{r_{\mathrm{UE}, i}}{t_{\mathrm{UE}, i}} x_{i} \\
& +\beta r_{\mathrm{UE}, i} \sum_{j=1, j \neq i}^{K}\left[\boldsymbol{W}_{\zeta}\right]_{i, j} \frac{1}{t_{\mathrm{UE}, j}} x_{j}+n_{i},
\end{aligned}
$$

and the SINR of the $i$ th UE is

$$
\gamma_{i}=\frac{\beta^{2} \cdot \rho \cdot\left|\frac{r_{\mathrm{UE}, i}}{t_{\mathrm{UE}, i}}\right|^{2} \cdot\left|\left[\boldsymbol{W}_{\zeta}\right]_{i, i}\right|^{2}}{\beta^{2} \cdot \rho \cdot\left|r_{\mathrm{UE}, i}\right|^{2} \cdot \sum_{j=1, j \neq i}^{N}\left|\left[\boldsymbol{W}_{\zeta}\right]_{i, j}\right|^{2}\left|\frac{1}{t_{\mathrm{UE}, j}}\right|^{2}+1},
$$

where $\rho=P / \sigma_{\mathrm{n}}^{2}$. Then the ergodic sum-rates of all UEs are

$$
\mathcal{E}[R]=\mathcal{E}\left[\sum_{i=1}^{K} R_{i}\right]=\mathcal{E}\left[\sum_{i=1}^{K} \log \left(1+\gamma_{i}\right)\right] .
$$

According to Eq. (29), it can be seen that only the calibration error causes the multi-user interference instead of the RF mismatches at the APs. Furthermore, we can mitigate the impact of the calibration error by increasing the transmitting power of the calibration signals. On the other hand, we can improve the calibration performance by using the TLS method with diversity combining, which will be shown in the following section.

\section{Simulation results}

System simulations have been carried out to illustrate the performance of our proposed calibration method. A single cell distributed large-scale MIMO system with zero-forcing downlink precoding is considered. For brevity of discussion and reproducibility of our results, we assume that there are 16 singleantenna APs uniformly on a circle of radius $200 \mathrm{~m}$ and four single-antenna UEs uniformly on a circle of radius $100 \mathrm{~m}$. The small-scale fading of both the transmission and calibration channels are supposed to follow a Rayleigh distribution, which has zero mean circularly symmetric complex Gaussian random variables with variance of $1 / 2$ per dimension. The path loss model is assumed as $c d^{-\eta}$, where $d$ is the distance between the transmitter and the receiver, $\eta=2$ is the path loss exponent, $c$ is the median of the mean path gain at the reference distance $d=1 \mathrm{~km}$. We define $\rho=c d^{-\eta} \cdot P / \sigma_{n}^{2}$ and $\rho_{\text {cal }}=$ $c d^{-\eta} \cdot P_{\text {cal }} / \sigma_{n}^{2}$ as the reference average SNR of the transmission signals and calibration signals, respectively. The amplitudes of the RF gains are assumed to be of log-normal distribution, and the phases are assumed to be of uniform distribution ${ }^{[8,10,11]}$. The variance of the amplitude mismatches and the range of the phase mismatches are set to be $\delta^{2}=2 \mathrm{~dB}$ and $\theta=\pi / 2$, respectively. 
Fig. 2 illustrates the performance of the sum-rates as a function of $\rho_{\text {cal }}$ when $\rho=20 \mathrm{~dB}$. It can be seen that all sum-rates increase with $\rho_{\text {cal }}$ and achieve a performance of perfect calibration. Moreover, with diversity gain, the system performance improves significantly, especially when $\rho_{\text {cal }}$ is in the low region.

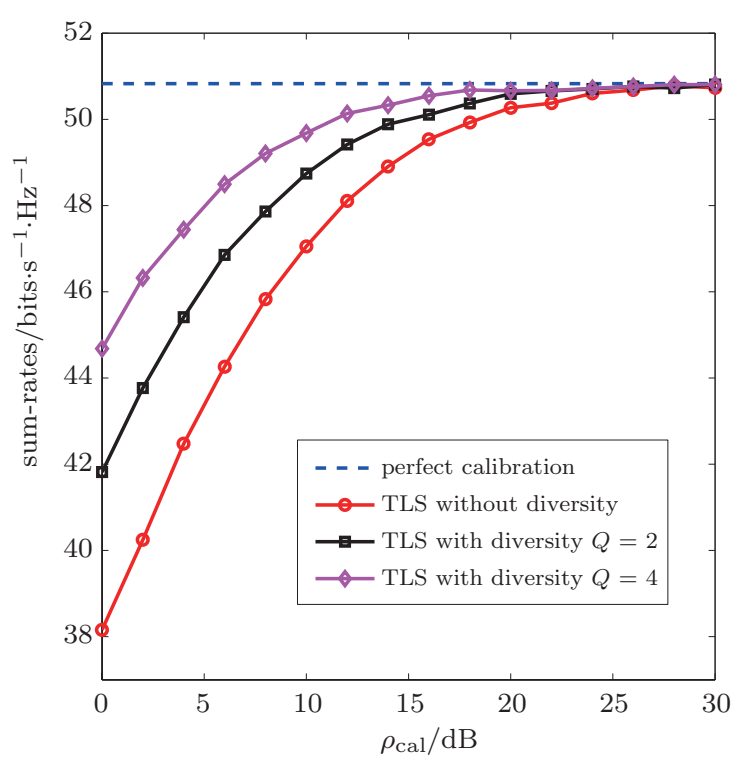

Figure 2 Sum-rates vs. $\rho_{\text {cal }}$

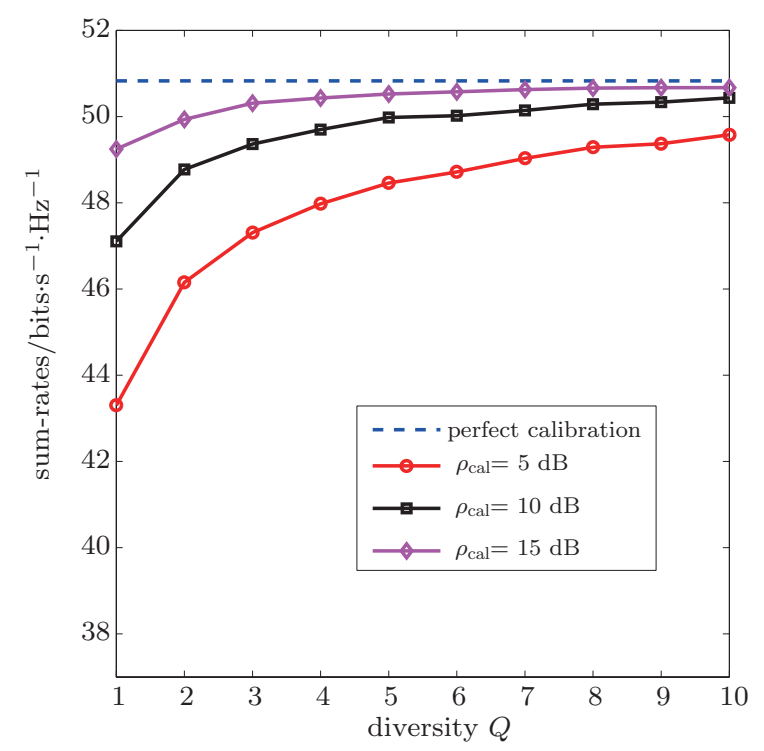

Figure 3 Sum-rates vs. diversity $Q$

Fig. 3 shows the relation between sum-rates and diversity $Q$ when $\rho=20 \mathrm{~dB}$. The average calibration SNR $\rho_{\text {cal }}$ is set to be $5 \mathrm{~dB}, 10 \mathrm{~dB}$, and $15 \mathrm{~dB}$ respectively. With an increase in $Q$, the sum-rates increase gradually but approach a performance limit that is lower than perfect calibration because of thermal noise. The performance with $\rho_{\text {cal }}=5 \mathrm{~dB}$ and $Q=8$ is the same as that with $\rho_{\text {cal }}=15 \mathrm{~dB}$. Thus, under low $\rho_{\text {cal }}$, we can achieve better calibration performance with a diversity gain.

\section{Conclusions}

In this paper, we investigated reciprocity calibration for distributed large-scale MIMO systems. In order to exclude UEs from the calibration procedure, the TLS method is extended to the case of partial calibration, where only the APs are involved in exchanging calibration signals. Furthermore, to overcome fluctuation in channels between APs, we proposed a scheme to obtain the diversity gain in order to improve the performance and robustness of the TLS method. Therefore, there exists a tradeoff between the transmitting power of the calibration signals and the diversity order.

\section{References}

[1] T. L. Marzetta. Noncooperative cellular wireless with unlimited numbers of base station antennas [J]. IEEE transactions on wireless communications, 2010, 9(11): 3590-3600.

[2] E. Larsson, O. Edfors, F. Tufvesson, et al. Massive MIMO for next generation wireless systems [J]. IEEE communications magazine, 2013, 52(2): 186-195.

[3] Z. Ma, Z. Q. Zhang, Z. G. Ding, et al. Key techniques for $5 \mathrm{G}$ wireless communications: network architecture, physical layer, and MAC layer perspectives [J]. Science China information sciences, 2015, 58(4): 041301.

[4] D. M. Wang, Z. L. Zhao, Y. Q. Huang, et al. Large-scale multi-user distributed antenna system for $5 \mathrm{G}$ wireless communications [C]//IEEE Vehicular Technology Conference, Glasgow, Scotland, 2015: 1-5.

[5] R. Zakhour, Z. Ho, D. Gesbert. Distributed beamforming coordination in multicell MIMO channels [C]//IEEE Vehicular Technology Conference, Barcelona, Spain, 2009: 1-5.

[6] H. Wei, D. M. Wang, H. L. Zhu, et al. Mutual coupling calibration for multiuser massive MIMO systems [J]. IEEE transactions wireless communications, 2016, 15(1): 606-619. 
[7] F. Kaltenberger, H. Jiang, M. Guillaud, et al. Relative channel reciprocity calibration in MIMO/TDD systems [C]//IEEE Future Network and Mobile Summit, Florence, Italy, 2010: 1-10.

[8] F. Huang, Y. Wang, J. Geng, et al. Antenna mismatch and calibration problem in coordinated multipoint transmission system [J]. IET communications, 2012, 6(3): 289-299.

[9] W. C. Zhang, H. Ren, C. H. Pan, et al. Large-scale antenna systems with UL/DL hardware mismatch: achievable rates analysis and calibration $[\mathrm{J}]$. IEEE transactions on communications, 2015, 63(4): 1216-1229.

[10] R. Rogalin, O. Bursalioglu, H. Papadopoulos, et al. Scalable synchronization and reciprocity calibration for distributed multiuser MIMO [J]. IEEE transactions on wireless communications, 2014, 13(4): 1815-1831.

[11] H. Wei, D. M. Wang, J. Z. Wang, et al. TDD reciprocity calibration for multi-user massive MIMO systems with iterative coordinate descent $[\mathrm{J}]$. Science China information sciences, 2016, 59(10): 102306.

[12] A. Bjorck, P. Heggernes, P. Matstoms. Methods for large scale total least squares problems [J]. SIAM journal on matrix analysis and applications, 2000, 22(2): 413-429.

[13] K. Nishimori, K. Cho, Y. Takatori, et al. Automatic calibration method using transmitting signals of an adaptive array for TDD systems [J]. IEEE transactions on vehicular technology, 2001, 50(6): 1636-1640.

\section{About the authors}

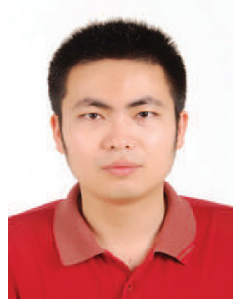

Hao Wei was born in 1988. He received the B.S. and the M.S. degrees from Nanjing University of Posts and Communications, Nanjing, China, in 2010, and 2013, respectively. He received the Ph.D. degree from Southeast University, Nanjing, China, in 2016. He is currently working for ZTE Corporation in Shanghai. His current research interests include massive MIMO, and reciprocity calibration. (Email: weihao@seu.edu.cn)

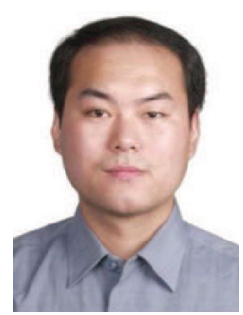

Dongming Wang [corresponding author] was born in 1977. He received the B.S. degree from Chongqing University of Posts and Telecommunications in 1999, the M.S. degree from Nanjing University of Posts and Telecommunications in 2002, and the Ph.D. degree from Southeast University in 2006. He joined the National Mobile Communications Research Laboratory at Southeast University, China, in 2006, where he has been an associate professor since 2010. His current research interests include turbo detection, channel estimation, distributed antenna systems, and large-scale MIMO systems. (Email: wangdm@seu.edu.cn)

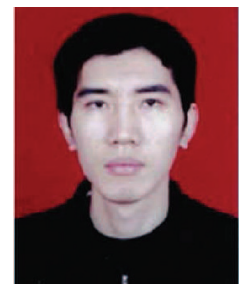

Jingyu Hua was born in 1978. He received his $\mathrm{Ph} . \mathrm{D}$. degree in radio engineering from Southeast University in 2006. That year, he joined Zhejiang University of Technology as an assistant professor, and is now a full professor. Dr. Hua has published more than 100 international journal and conference papers. His research interests lie in the area of parameter estimation, channel modeling, wireless localization and digital filtering in mobile communications. (Email: eehjy@zjut.edu.cn)

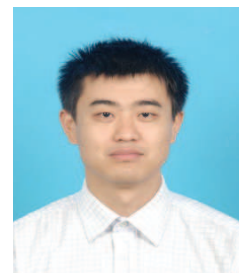

Junhui Zhao was born in 1973. He received his Ph.D. degree from Southeast University in 2004. He is currently a professor in the School of Electronic and Information Engineering, Beijing Jiaotong University, Beijing, China. He is also a senior member of the Institute of Electrical and Electronics Engineers (IEEE). Prof. Zhao has published over 50 papers and more than 10 patents pending. His current research is wireless location and cognitive radio. (Email: jhzhao@ieee.org) 